\title{
Kniest Dysplasia. A Histochemical Study of the Growth Plate
}

\author{
WILLIAM A. HORTON AND DAVID L. RIMOIN \\ Division of Medical Genetics, Harbor-UCLA Medical Center, Torrance, California USA
}

\begin{abstract}
Summary
Chondro-osseous tissue from four patients with the Kniest dysplasia was studied histochemically using a new plastic embedding technique. Extensive vacuolar changes were observed $p-1$ throughout the endochondral growth plate and adjacent resting cartilage. These changes occurred within the cartilage matrix and also in the lacunae of degenerating chrondrocytes. The septa of the lesions contained chondroitin sulfate, but little keratan sulfate or collagen. Resting cartilage not adjacent to the growth plate stained irregularly and showed few of the vacuolar lesions, and chondrocytes were enlarged and contained cytoplasic inclusions, but no vacuolar material. Thus, there appears to be a sequence of events initiated by cellular accumulation of a substance and progressing to cellular and matrix degeneration.
\end{abstract}

\section{Speculation}

The findings suggest that there may be a defect in the synthesis, structure, or secretion of a major cartilage matrix component (e.g., proteoglycan, collagen) which leads to its accumulation in the chondrocyte rough endoplasmic reticulum. Both cellular and matrix degeneration subsequently occur either due to a toxic effect of this material or to the absence of the normal molecule.

The Kniest dysplasia (previously termed Kniest syndrome. The name was changed at the Second International Conference for Nomenclature for Constitutional Disease of Bone, Paris, 1977) is a generalized disorder of connective tissue characterized by short trunked dwarfism, flat round facies, cleft palate, myopia, and joint limitation (9). Because of the multisystem involvement, a defect in a common connective tissue component, such as collagen, proteoglycan or glycosaminoglycan (GAG), must be considered. Standard histologic evaluation of the growth plate tissue obtained from patients with this disorder has demonstrated a characteristic histopathologic picture named "Swiss Cheese Cartilage", because of the numerous empty spaces lying within the resting cartilage (9-11). Ultrastructural studies have shown that the resting chondrocytes are filled with dilated cisternae of rough endoplasmic reticulum, and as well, there are irregular aggregates of collagen in the resting cartilage matrix $(14,16)$. Stanescu and Maroteaux (15) have recently demonstrated an electrophoretic abnormality in proteoglycans in this disorder and have postulated a defect in the synthesis or transfer of these molecules. Despite these studies, the specific pathogenetic mechanisms responsible for the manifestations of this disorder remain poorly understood.

A new histochemical approach using plastic embedding has recently been developed to study growth plate tissue (5). Many of the steps of standard histologic processing which damage tissue (e.g., decalcification) are eliminated so that much more information can be obtained about both the structural and the biochemical organization of the growth plate. In particular, the major growth plate components such as collagen, proteoglycan, and calcium, can be identified and semiquantitated. We have studied chondroosseous tissue obtained from four patients with the Kniest dysplasia in this fashion, and as a result, have gained further insights into the basic underlying defect in this disorder.

\section{METHODS}

Rib and iliac crest biopsies were obtained from four patients with well documented Kniest dysplasia after appropriate consent. Their ages ranged from 8-23 yr, and three patients were prepubertal. At the time of the procedure, a portion of each biopsy was fixed and processed by standard methods for routine histology. Another portion was rapidly frozen in liquid nitrogen and stored at $-70^{\circ} \mathrm{C}$. Recently, when the plastic embedding technique became feasible (1), a 4-5 $\mathrm{mm}$ slice of tissue representative of the biopsy was cut at $-40^{\circ} \mathrm{C}$ and dehydrated at that temperature in a solution of $70 \%$ ethylene glycol and $30 \%$ formol-calcium (10\% formalin, $2 \%$ calcium acetate) for 4 days. The temperature was then raised to $-10^{\circ} \mathrm{C}$ and dehydration completed over the next 24 $\mathrm{hr}$ in 100\% ethylene glycol. The slices were then infiltrated in the monomeric form of glycol methacrylate (GMA, Polysciences) at $4^{\circ} \mathrm{C}$. After four changes at 12 -hr intervals, polymerization was carried out over a 12 -hr period at $4^{\circ} \mathrm{C}$. One micron sections were cut on a Sorvall JB-4 microtome with a $1 / 2$-in knife and sections were stained with a series of staining techniques modified from existing methods particularly for GMA embedded cartilage tissue. These included staining for $15 \mathrm{~min}$ in a $1 \%$ alcian blue in $0.4 \mathrm{M}$ and $0.9 M \mathrm{MgCl}_{2}(12,13)$ as well as after digestion by $0.1 \%$ testicular hyaluronidase (4), and counterstaining in $1 \times 10^{-3} M$ basic fuchsin buffered at $\mathrm{pH} 7.4$ for $1 \mathrm{~min}(1)$, and "stains-all" (1ethyl-2-\{3-(1-ethylnaphtha $\{1,2 \mathrm{~d}\}$ thiazolin-2-ylidene)-2-methylpropenyl $\}$-naphtho $\{1,2 \mathrm{~d}\}$ thiazolium bromide $)(0.1 \%$ stains-all in $100 \%$ foramide $7 \mathrm{ml}, 5 \times 10^{-3} M$ veronal buffer $\mathrm{pH} 4.32 \mathrm{ml}, \mathrm{H}_{2} \mathrm{O}$ $40 \mathrm{ml}$ ) (3), to characterize the distribution of proteoglycan and the type of bound glycosaminoglycan (chondroitin sulfates or keratan sulfate); a trichrome stain consisting of $0.9 \%$ Biebrich scarlet $/ 0.1 \%$ acid fuchsin $1 \%$ acetic acid for $5 \mathrm{~min}(8)$ and $0.5 \%$ aniline blue/ $0.06 \mathrm{M} \mathrm{HCl}$ for $5 \mathrm{~min}(7)$ combined with the von $\mathrm{Kossa}\left(\mathrm{AgNO}_{3}\right)$ method (2) to study intracellular morphology and matrix calcification; and the Van Gieson stain (7) and a silver methenamine technique (8) to evaluate collagen distribution. Age-matched controls were processed and stained in a similar fashion.

\section{RESULTS}

Similar changes were observed in all specimens studied (because of her age and closed epiphyses, no growth plate was present in tissue from the 23-yr-old). Throughout the growth plate and resting cartilage, especially that adjacent to the growth plate, were scattered large areas of abnormal matrix composed of multiple vacuoles of varying size lying within a delicate network of thin septa (Fig. 1). Within the lesions were numerous large degenerating chondrocytes lying in lacunae that were filled with vacuolar material identical in appearance to the extralacunar material already described (Fig. 2a). It could not be determined if this 


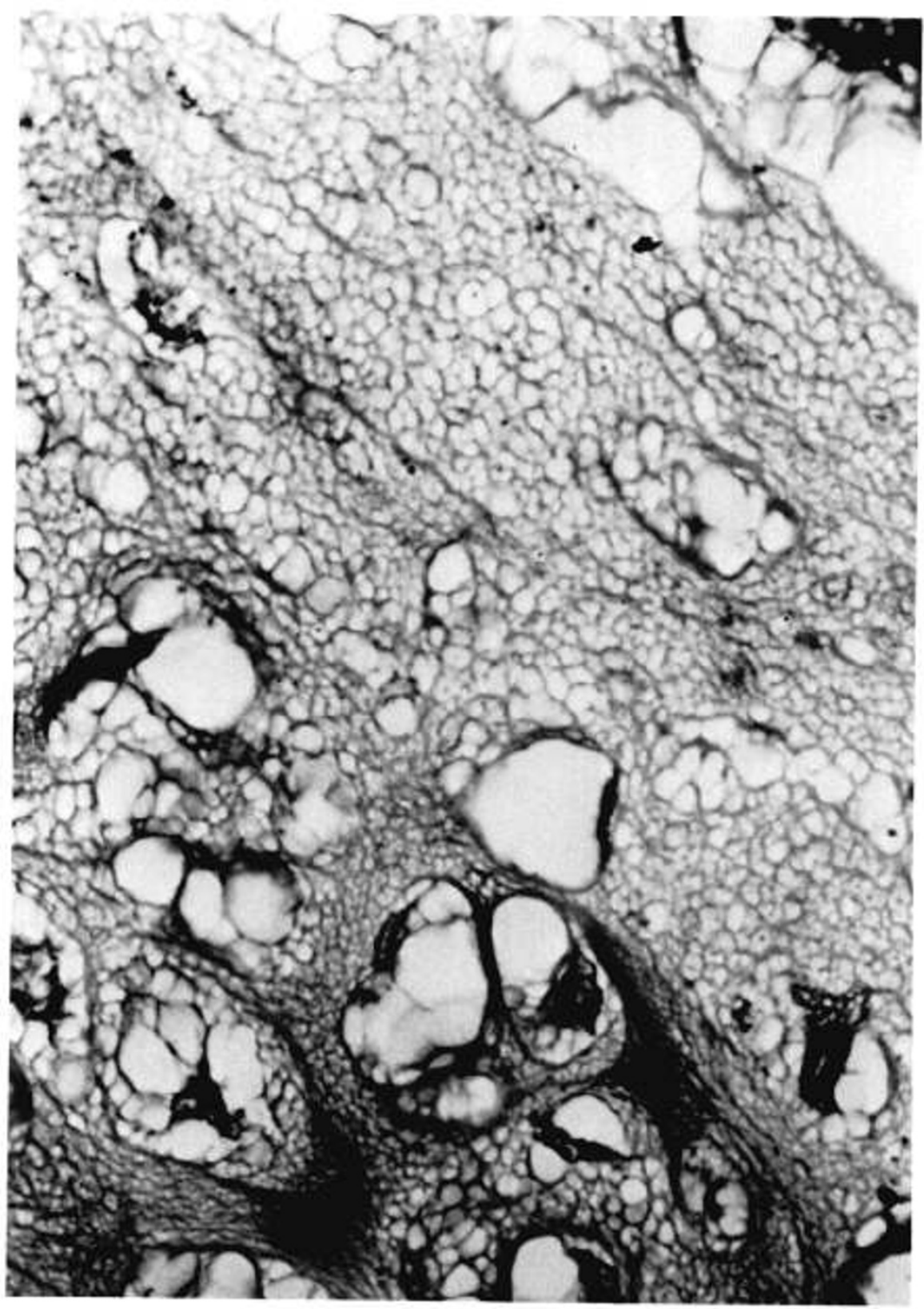

Fig. 1. Cartilage tissue from a 12-yr-old female with the Kniest dysplasia. Note the vacuolar degeneration of extralacunar matrix in area of resting cartilage adjacent to the growth plate. Alcian blue/basic fuchsin, $960 \mathrm{X}$.

vacuolar material was intracellular or extracellular-intralacunar. Although much more extensive, it did resemble the material that accumulates between the withdrawing cell membrane and lacunar border of chondrocytes in the degenerative zone of normal cartilage (Figure 2b). In some areas, the septa were lost resulting in the formation of large Swiss Cheese-like vacuoles. The septa stained positively with alcian blue at $0.4 M \mathrm{MgCl}_{2}$, but staining was lost both when the concentration was raised to $0.9 M$ and after hyaluronidase digestion, suggesting that the GAG is predominantly chondroitin sulfate rather than keratan sulfate (keratan sulfate continues to stain after both maneuvers). Control cartilage matrix however showed similar staining for keratan sulfate by these techniques. The septa did not stain positively with prolonged incubation in silver methenamine, a technique in which collagen fibers appear to be impregnated by silver. There was, however, irregular staining along the edge of the lesions.

Despite the degeneration of cells and matrix already described, the chondrocytes within the growth plate per se did form columns (Fig. 3). Cellular development within the adjacent columns, how- ever, was poorly synchronized and metaphyseal vascular invasion was very irregular. The matrix septa were wider than normal and often filled with the vacuolar material. The calcification of the cartilage septa at the chondro-osseous junction (provisional calcification) as well as the calcification of bone matrix appeared normal.

In the resting cartilage, beyond that bordering the growth plate, the vacuolar lesions described above were smaller in size and less frequent. The matrix stained normally by most stains, but consistently stained poorly with the silver methenamine method. Resting chrondrocytes, however, were larger than normal, irregularly shaped, and contained cytoplasmic inclusions which did not stain with alcian blue (Fig. 4).

\section{DISCUSSION}

The Kniest dysplasia has been called the Swiss Cheese cartilage syndrome because of the characteristic histologic appearance of numerous empty spaces lying within the resting cartilage matrix 




Fig. 2. Growth plate specimen showing hypertrophic and degenerating chondrocytes: $A$ from 12 -yr-old female with the Kniest dysplasia, stains all $960 \mathrm{X}$ and $B$ normal control, alcian blue/basic fuchsin, $1000 \mathrm{X}$. Note that the vacuoles within the lacunae of the Kniest cells resemble the extralacunar material seen in Figure 1 and also the vacuolar changes which occur normally in degenerating chrondrocytes (arrows).

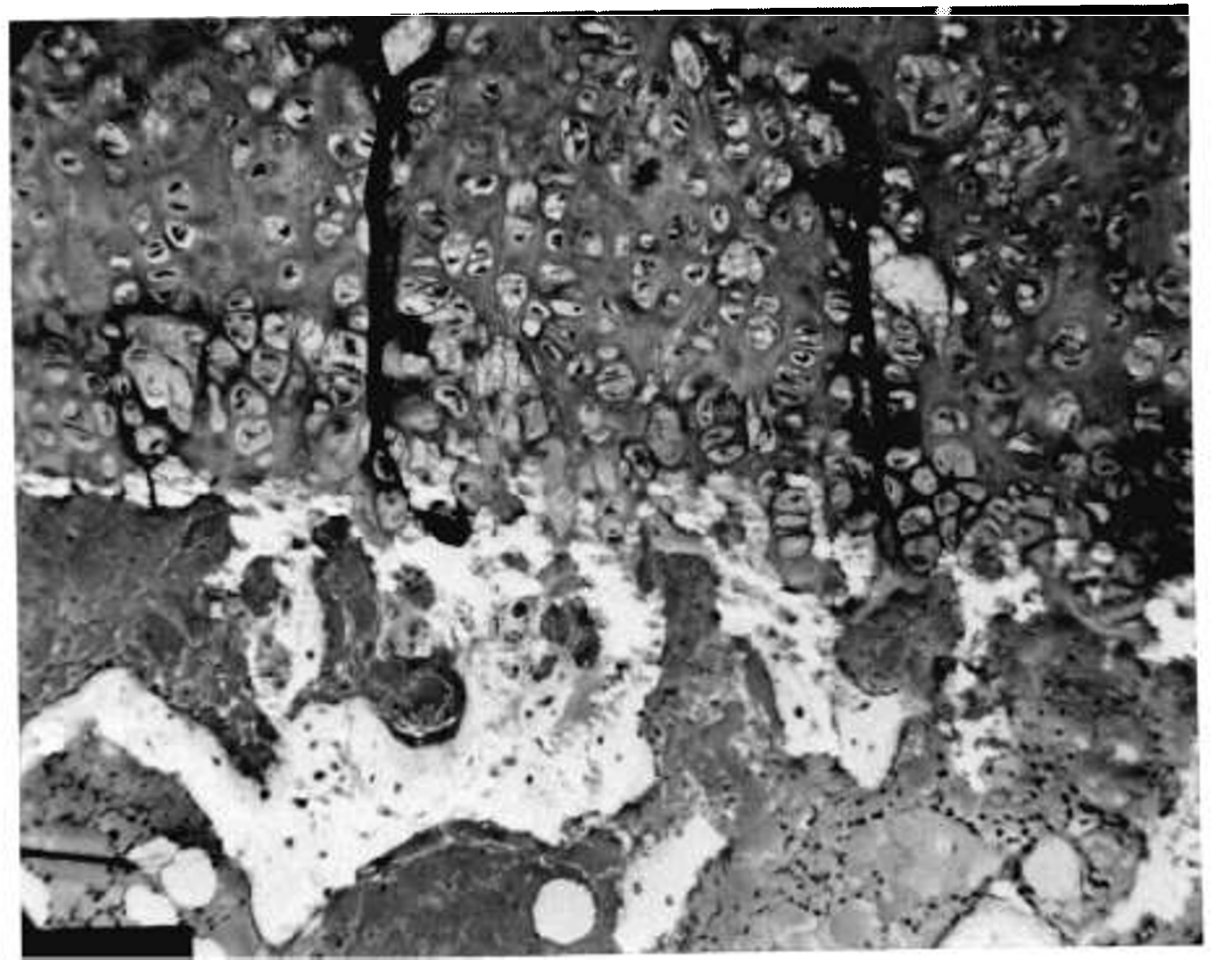

Fig. 3. Growth plate from 12-yr-old female with the Kniest dysplasia. Note irregular column formation and dysynchronous cell maturation. Cartilage above, bone below (appears white due to lack of staining). Alcian blue/basic fuchsin, $100 \mathrm{X}$. 


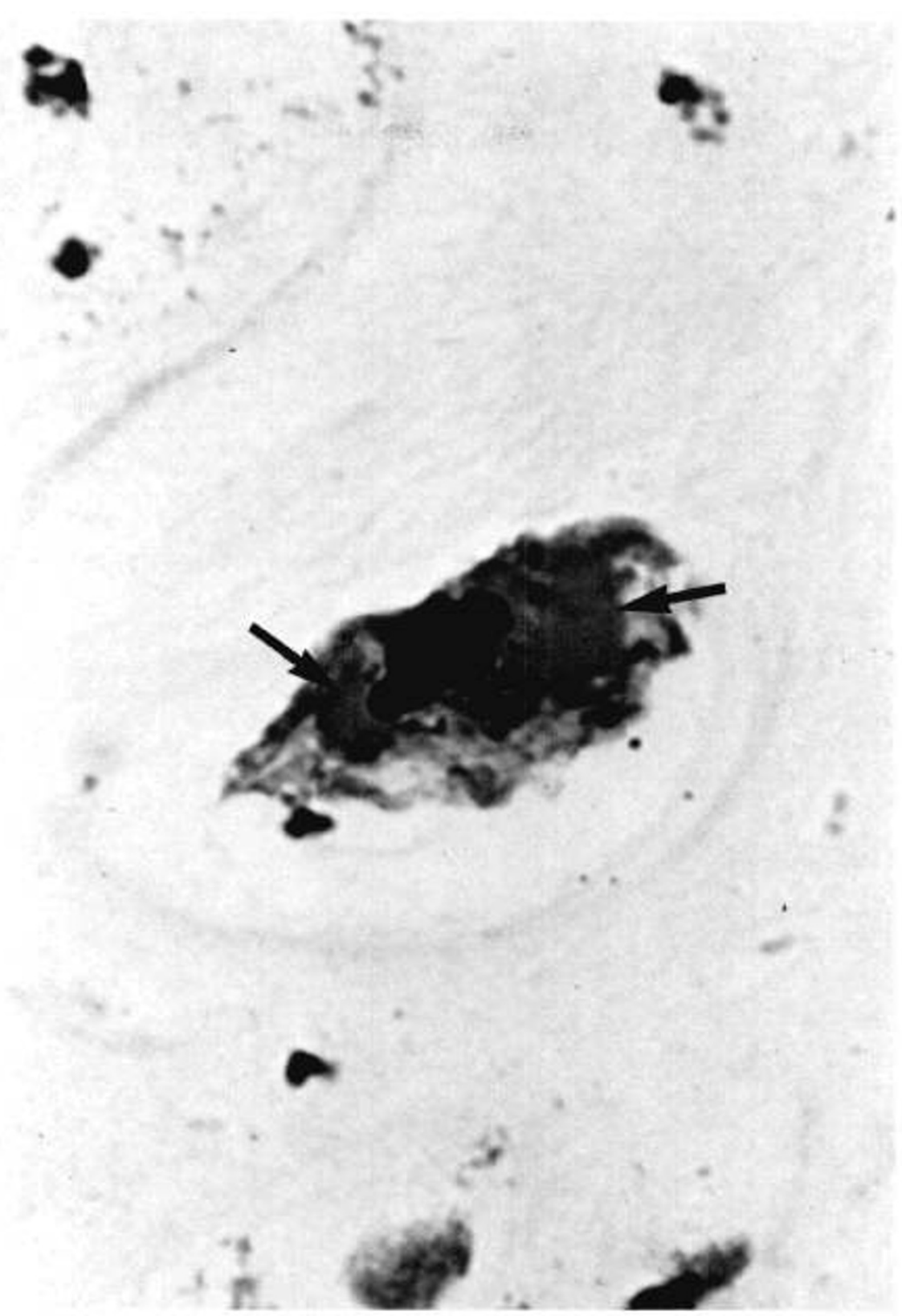

Fig. 4. Resting chondrocyte from 12-yr-old female with Kniest dysplasia. Note cytoplasmic inclusion (arrows). Tri-chrome stain, $2400 \mathrm{X}$.

(9). The plastic embedding technique employed in this study revealed that these vacuolar lesions are much more extensive than previously demonstrated with paraffin embedded tissues and involve not only resting cartilage matrix, but the matrix septa within the growth plate as well. Moreover, vacuolar material identical in appearance to that found in the matrix lesions was observed within the lacunae of degenerating cells. Although these vacuoles resemble those seen in the lacunae of normal degenerating chondrocytes, the vacuolar contents have not been characterized histochemically in either case (see later in this paper) and might well differ. In areas where the matrix appears more intact, there is no such intralacunar material. Instead, chondrocytes contain cytoplasmic inclusions which have been previously shown to be dilated cisternae of endoplasmic reticulum $(14,16)$.

The process occurring within this cartilage can be partially characterized histochemically. Although the contents of the vacuoles cannot be identified because of loss, presumably during tissue preparation, the septa of the lesions contain large amounts of proteoglycan that is composed of predominantly chondroitin sulfate, as was control cartilage matrix, and little or no organized collagen. This is different from other disorders manifesting matrix degeneration such as diastrophic dwarfism in which the fibrous component of the matrix lesion is composed of thickened collagen fibrils (5). Except for the irregular staining along the edges of the matrix lesions, collagen staining throughout the resting cartilage was consistently greatly reduced compared to normal. Presumably, this could be due to an absolute decrease in the amount of matrix collagen or to an abnormality that interferes with the normal staining interaction, such as structural abnormality in collagen or in some other matrix molecule which interacts with collagen, i.e., proteoglycan.

It is impossible to draw any conclusions about the specific pathogenetic process responsible for these findings. One can speculate, however, that a defect in the structure, synthesis, or secretion of a matrix component leads to its accumulation within the rough endoplasmic reticulum. This could then produce cell toxicity and premature degeneration of the chondrocytes. Matrix degeneration could occur secondary to chondrocyte degeneration with the accumulation of vacuolar material within acellular spaces, or alternatively, secondary to the absence within the matrix of an essential component. Thus, although the actual process is unclear, there does appear to be a sequence of events initiated by the cellular accumulation of a substance and progressing to degeneration of both cells and matrix. The recent demonstration of 
electrophoretic abnormalities of cartilage proteoglycan in this disorder (15) suggests that the basic defects in the Kniest dysplasia may be in this macromolecule. However, defects of other matrix components can not be excluded. Additional histochemical and biochemical studies will be needed to further characterize the specific defect.

\section{REFERENCES AND NOTES}

1. Bennett, H. W., Wyrick, A. D., Lee, S. W. and McNeil, J. H.: Science and art in preparing tissues embedded in plastic for light microscopy with special reference to glycol methacrylate, glass knives, and simple stains. Stain Technol., 51: $71(1976)$.

2. Culling, C. F. A.: In: Handbook of Histopathological and Histochemical Techniques. p. 470 (Butterworths, Toronto, 1974).

3. Green, M. R., and Postewka, J. V.: Simultaneous differential staining by a catatonic carbocyanine dye of nucleic acids, proteins, and conjugated proteins, II. Carbohydrate and sulfated carbohydrate-containing proteins. J. Histochem. Cytochem., 22: 774 (1974).

4. Hirshman, A., and McCabe, D. M.: The effect of proteolytic enzymes and hyaluronidase on the intracellular $\beta$ and $\gamma$ metachromatic granules and the matrix of rat epiphyseal cartilage. Anat. Rec., 180: 617 (1974).

5. Horton, W. A., and Rimoin, D. L.: Histochemical characterization of the endochondral growth plate, a new approach to the study of the chondrodystrophies. Birth Defects. Original Article Series (In Press).

6. Kim. H. G., Beratis, N. G., Brill, P., Raab, E., Hirschhorn, K., and Matalon, R.: Kniest syndrome with dominant inheritance and mucopolysacchariduria. Amer. J. Human Genet.. 27: 755 (1975).

7. Lillie. R. D., and Fullmer, H. M.: In: Histopathologic Technique and Practical Histochemistry. pp. 679, 702 (McGraw-Hill, New York, 1976).

8. Luna, L. G.: In: Manual of Histologic Staining Methods of the Armed Forces
Institute of Pathology. pp. 94, 97 (McGraw-Hill, New York, 1968).

9. Rimoin, D. L.: The chondrodystrophies. Adv. Hum. Genet., 5: 1 (1975).

10. Rimoin, D. L., Hollister, D. W., Lachman, R. S., Kaufman, R. L., McAlister, W. H., Rosenthal, R. E., and Hughes, G. N. F.: Histologic studies in the chondrodystrophies, In: D. Bergsma; Skeletal Dysplasias, Birth Defects: Original Article Series, 10: 274 (1974).

11. Rimoin, D. L., Silberberg, R., and Hollister, D. W.: Chondro-osseous pathology in the chondrodystrophies. Clin. Orthop., (114): 137 (1976).

12. Scott, J. E., and Dorling, J.: Differential staining of acid glycosaminoglycans (mucopolysaccharides) by alcian blue in salt solutions. Histochemie, 5: 221 (1965).

13. Scott, J. E., Dorling, J., and Stockwell, R. A.: Reversal of protein blocking of basophilia in salt solutions, implications in the localization of polyanions using alcian blue. J. Histochem. Cytochem., 16: 383 (1968).

14. Silberberg, R.: Ultrastructure of cartilage in chondrodysplasias. Birth Defects Original Article Series, 10: (12) 306 (1974)

15. Stanescu, V., and Maroteaux, P.: Gel electrophoretic studies on proteoglycans and collagen of abnormal human growth cartilage: proteoglycan abnormalities in pseudoachondroplasia in Kniest disease. Pediatr. Res., 9: 779 (1975).

16. Stanescu, V., Stanescu, R., and Maroteaux, P.: Etude morphologique et biochemique du cartilage de crossiance dans les osteochondrodysplasias. Arch. Franc. Pediat., 34(Suppl 3): 48 (1977).

17. The authors thank Ms. Carmen Carpentier for technical assistance.

18. The present address of Dr. William A. Horton is: Division of Metabolism Endocrinology and Genetics, Kansas University Medical Center, Kansas City, Kansas, USA.

19. This research was supported in part, by United States Public Health Service Research grant HD 11966, Clinical Research Center grant RRO 0425, Graduate Research Training grant GM 07414, and a Research Birth Defects Center Grant from the National Foundation-March of Dimes.

20. Received for publication November 21,1977

21. Accepted for publication October 12, 1978 